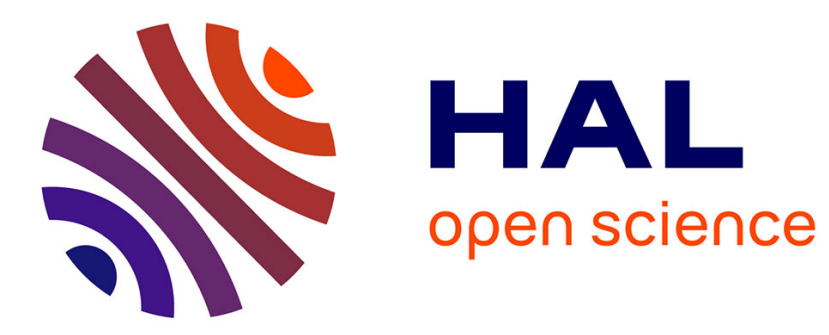

\title{
A numerical method to optimize the design of a space inflatable membrane reflector
}

\author{
Rabah Bouzidi, Yann Lecieux
}

\section{To cite this version:}

Rabah Bouzidi, Yann Lecieux. A numerical method to optimize the design of a space inflatable membrane reflector. Acta Astronautica, 2011, 74, pp.69-78. 10.1016/j.actaastro.2011.12.009 . hal01004922

\section{HAL Id: hal-01004922 \\ https://hal.science/hal-01004922}

Submitted on 3 Dec 2016

HAL is a multi-disciplinary open access archive for the deposit and dissemination of scientific research documents, whether they are published or not. The documents may come from teaching and research institutions in France or abroad, or from public or private research centers.
L'archive ouverte pluridisciplinaire HAL, est destinée au dépôt et à la diffusion de documents scientifiques de niveau recherche, publiés ou non, émanant des établissements d'enseignement et de recherche français ou étrangers, des laboratoires publics ou privés. 


\title{
A numerical method to optimize the design of a space inflatable membrane reflector
}

\author{
Rabah Bouzidi, Yann Lecieux \\ L'UNAM Université, Université de Nantes-Ecole Centrale Nantes, GeM, Institut de Recherche en Génie Civil et Mécanique, UMR CNRS 6183, 2 rue de la Houssinière, \\ BP 9220844322 Nantes cedex 3, France
}

\begin{abstract}
A numerical method is proposed to optimize the design of a space inflatable membrane reflector. The initial geometry is expressed by polynomial series weighted by a set of shape parameters. The problem is formulated as a minimization of a cost function representing the difference between the effective shape of the reflector and a perfect parabolic surface. The minimization is performed using the Nelder-Mead method or downhill simplex method. The cost function is computed at each vertex of a simplex defined in the space of optimization parameters by solving direct problem thanks to a finite element method. The finite element model handles geometrical non-linearities and takes into account phenomena like membrane wrinkling and torus buckling which may affect the reflector shape when inflated.
\end{abstract}

Keywords:Design, Membrane Inflatable antenna, Gossamer structures, Wrinkling, Energy minimization, Initial form finding

\section{Introduction}

The interest of using large spacecraft structures (expandable or inflatable) involving thin film surface has been identified since the 1950s. The first inflatable space structures were the communication balloons of the Echo series. The larger one was a $30 \mathrm{~m}$ diameter balloon deployed using inflation gas. Since, technology developments have been made to demonstrate the potential of space inflatable structures [1,2]. Then in 1996 the Jet Propulsion Laboratory (JPL) performed the first experiment of deploying in space an inflatable lenticular antenna [3,4]. The structure is a $14 \mathrm{~m}$ parabolic reflector supported around its perimeter by an inflatable torus. The whole framework was attached to a Spartan satellite by the means of three $28 \mathrm{~m}$ inflatable struts (see Fig. 1). In this paper we propose a method to design optimally this kind of reflector that could be used as solar concentrator or radiometer system.

Compared to traditional spacecraft structures, these gossamer structures could provide many advantages such as reduced mass and package volume. However several serious difficulties limit their massive development:

- The finding of the neutral shape of the non-stressed membrane (non-pressurized structure).

- The choice of the pressure level in the different parts of the structure.

- The definition of the inflation technique for controllable deployment.

- The rigidization mechanism.

Even if the design optimization (pressure and shape) of the inflatable antenna is on current technological interest, only few works have been published on the topic [5]. Some applications (such as solar reflectors) require to obtain an inflatable parabolic shape of good accuracy. Analytical models can be found in the literature to approximate the 


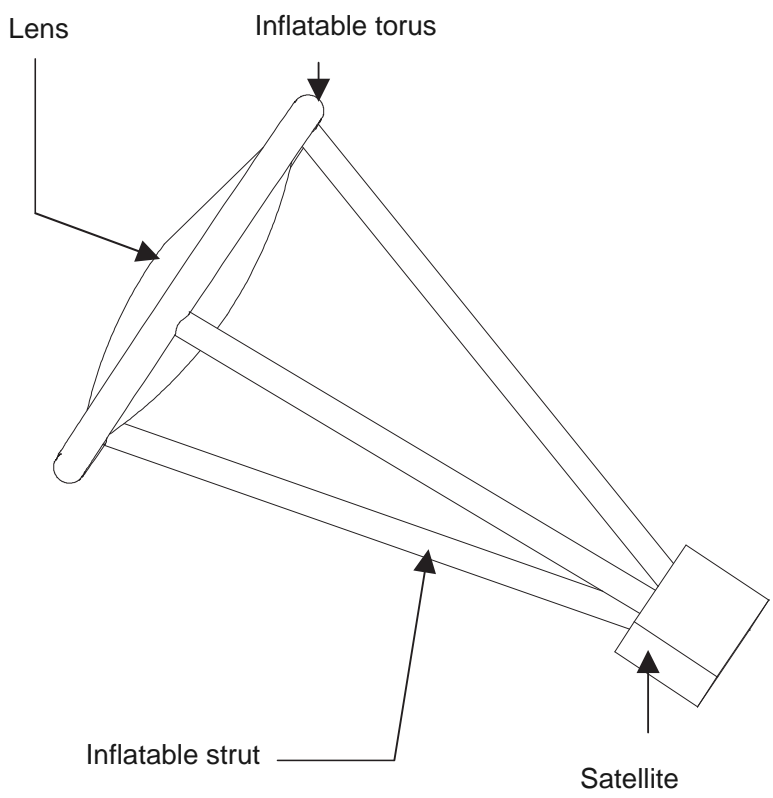

Fig. 1. Inflatable parabolic circular antenna.

deformed shape of a circular membrane clamped at the rim and subjected to uniform pressure. The first one was published by Hencky in the 1910s [6] and remains of interest today. Since, subsequent contributions have been brought $[7,1]$ but are generally restricted to initially flat membranes. The problem studied here involves the coupling between the deformations in the torus and in the reflector, large deformations and the possible wrinkling of the membrane. Even if the analytical solutions are more attractive means than the finite element models, in the present case it seems to be not realistic to built such solutions.

This paper is concerned with initial form finding and load optimization of an inflatable parabolic reflector with an efficient inverse method.

The initial shape of the antenna is represented by a series of parameters defining an axisymmetric shape of the lenticular reflector. We also considered the inflating pressure as an optimizing parameter. The inverse method uses a simplex defined in the parameters space and needs the evaluation of the cost function (RMSE) at each vertex of the simplex. The Nelder-Mead method or downhill simplex method is used to find a local minimum of the RMSE function. The RMSE computation is done thanks to a robust finite element model which is the key point of the procedure.

Indeed, the simulation of inflatable membrane structures having very small bending stiffness is a very challenging task. This is essentially due to the highly non-linear nature of the involved phenomena, including large deflection, large rotation and followers pressure. Moreover some instabilities, such as torus buckling and membrane wrinkling, might occur leading to unwanted shapes.

Over the last years, significant contributions to the non-linear analysis including buckling have been brought.
Nevertheless, the simulation of membrane wrinkling remains difficult and consequently a current research area. It is obvious that this phenomenon must be considered in order to compute the deformed shape of the reflector and therefore be able to accurately evaluate the RMSE function. It is essential to take into account all these specificities of lightweight structures in order to expect sufficient precision of the deployed structure.

Here the finite element analysis is performed by using a true membrane element. The numerical solution of the direct problem is carried out by direct minimization of the total potential energy. This is done by the means of an iterative method such as the conjugate gradient. Although the proposed approach is theoretically equivalent to the traditional finite element method, it proves to be an attractive alternative which is particularly efficient for thin wrinkled structures. The main interest is that no specific buckling analysis is carried out during the solving procedure which is well adapted to the case of an optimization process.

The inverse problem is based on zero-order minimization method of Nelder-Mead which does not needs the gradient of the cost function. The zero-order methods have the advantage that only cost function evaluation is needed in the minimization process.

The paper is organized as follows: Section 2 presents the aim and scope of the study. Section 3 proposes a parameterization of the reflector shape. In Section 4, the finite element model is described and the minimization algorithm is discussed. The results of the study and validation tests are presented in Section 5, then Section 6 concludes the paper.

\section{Aim and scope}

The aim of this study is to design a reflector of a $2 \mathrm{~m}$ diameter according to the material data and the geometry depicted in Table 1 . The structure is formed of two parts pressurized at different levels: the lens and the torus.

The reflector is similar to the IAE experiment $[3,4]$ with different dimensions. It is made with a $6.3 \mu \mathrm{m}$ film. The lens is supported around its perimeter by a $0.2 \mathrm{~m}$ diameter torus made with a $300 \mu \mathrm{m}$ neoprene coated Kevlar. The material and the diameter of the torus could be optimization parameters. Nevertheless in this study the choice was made to fix their values.

The problem to solve is formulated as follow: What are the pressures (in the reflector and in the torus) and the

Table 1

Geometry and material data.

\begin{tabular}{lll}
\hline Data & Lens & Torus \\
\hline Young modulus $(\mathrm{GPa})$ & 6.0 & 15 \\
Poisson ratio & 0.3 & 0.3 \\
Thickness $(\mu \mathrm{m})$ & 6.3 & 300 \\
Radius $(\mathrm{m})$ & 1 & 0.1 \\
Focal length & $1 \mathrm{~m}$ & \\
\hline
\end{tabular}


neutral shape which lead to a perfect parabolic shape for the reflector once inflated?

The set of $m$ optimizing parameters $\{p\}$ includes the initial shape coefficients, noted $\alpha_{i}$, and also the pressures in the reflector and in the torus supporting structure. The initial shape coefficients define the neutral shape of the reflective surface. The shape of the reflector $z(\{p\})$ after inflation is thus a function of $\{p\}$. The RMSE, which quantifies the gap between $z(\{p\})$ and the target parabolic shape $z_{t h}$, is computed. The previous problem becomes a minimization study whose aim is to find the set of parameters $\{p\}$ which minimize the function RMSE $(\{p\})$.

The function $R M S E(\{p\})$ is highly nonlinear which might include bifurcated solutions. It is then very difficult to built its analytical derivatives with respect to $\{p\}$. Its minimization is achieved by zero-order downhill method. The simplex method of Nelder-Mead [8] has been used here. This method consists of evolving a simplex of $m+1$ vertices, in the optimizing parameters space of $m$ dimensions. This iterative process is repeated until the simplex size becomes inferior to an objective value $\varepsilon$. This method requires only to evaluate the cost function RMSE values at the simplex vertices and leads to a local optimum. The RMSE evaluation is achieved using a finite element analysis. The flowchart of the optimization procedure is shown in Fig. 2.

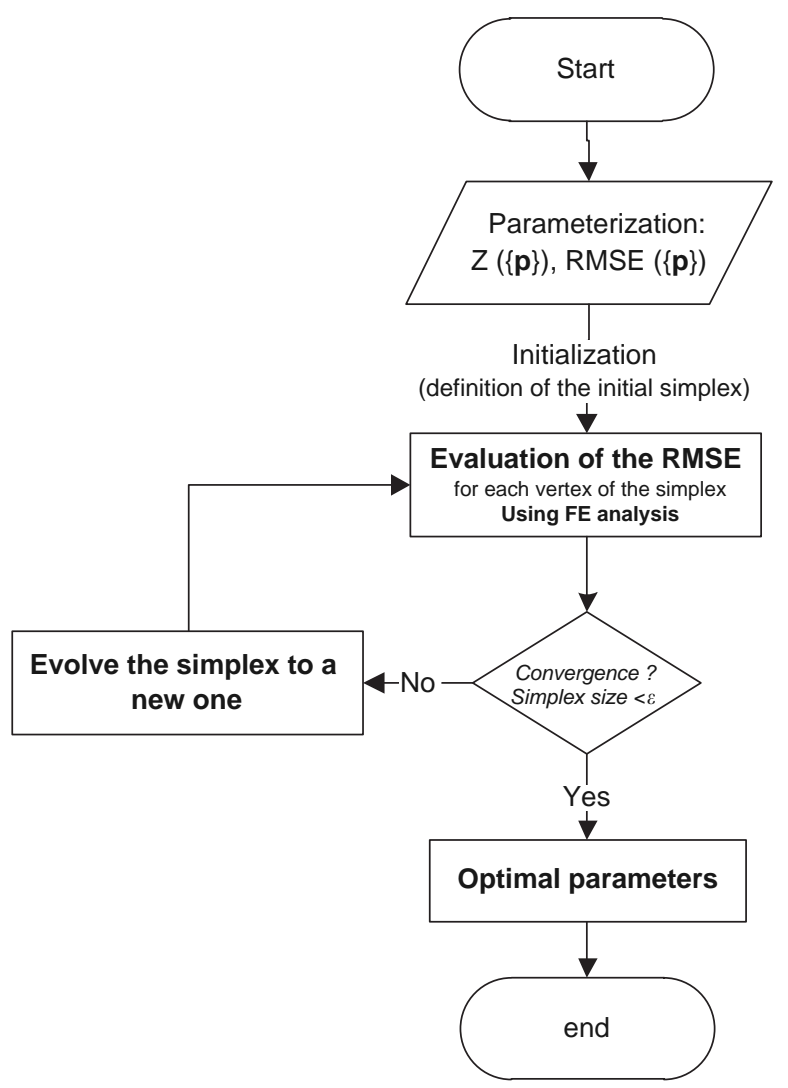

Fig. 2. Flowchart of the optimization procedure.

\section{Optimization of the shape reflector}

In this section, we discuss the representation of the initial shape of the lens. It corresponds to the configuration for which the membrane is not stressed and defines the geometry to be designed.

\subsection{Formulation of the cost function}

The deformed shape, due to the internal pressure, should be as close as possible of a perfect parabola defined in the expression (1) giving the deflection from the median plane

$z_{\text {th }}(\rho)=\frac{R^{2}}{4 f}\left(1-\rho^{2}\right)$

The formulation of the objective function $z_{\text {th }}$ depends on the lens radius $R$, the focal length $f$, and the adimensional radius $\rho=r / R$.

The initial deflection $Z$ of the neutral shape i.e. without pressurization is defined using an odd power series polynomial expansion such as

$Z=\frac{R^{2}}{4 f} \sum_{i=1}^{n} \alpha_{i}\left(1-\rho^{2 i}\right)$

Using this formulation, the initial shape is defined only with a finite number of shape parameters $\{\alpha\}=\alpha_{1}, \ldots, \alpha_{n}$. This is an arbitrary parameterization which is used to guarantee the axial symmetry of the reflector.

The optimization target becomes the minimization problem of a function quantifying the gap between the pressurized shape and the perfect parabola whose deflection are respectively $z$ and $z_{\text {th }}$ (see Fig. 3). This function is the RMSE (Root Mean Square Error) written in Eq. (3)

$R M S E=\sqrt{\frac{1}{n} \sum_{i=1}^{n}\left(z^{i}-z_{t h}^{i}\right)^{2}}$

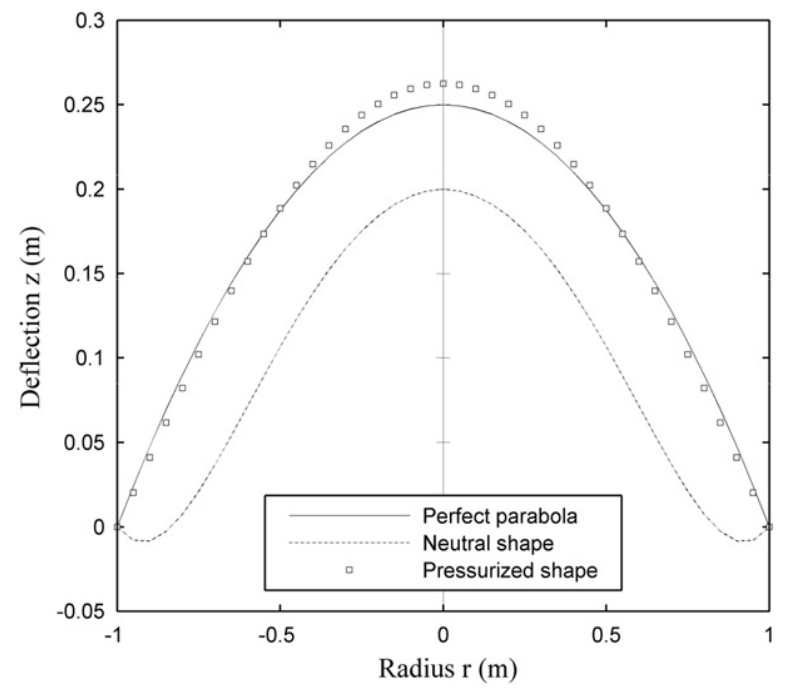

Fig. 3. Neutral, pressurized and perfect shape of the lens. 
The deflection difference $z^{i}-z_{t h}^{i}$ is evaluated for each vertex $i$ of the whole mesh vertices $n$ of the discretized shape of the lens.

\subsection{Optimizing parameters}

A pertinent choice of the optimizing parameters requires the identification of the physical parameters having an influence on the value of the RMSE i.e. on the deformed shape. Among these influent parameters we can list the torus pressure $P_{t}$, the lens pressure $P_{l}$, the material data (Young modulus and Poisson ratio) and the coefficients $\alpha_{i}$. Technological constraints have led to fix the material data.

As first set, the following optimizing parameters can be selected $\{p\}=\left\{P_{t}, P_{l}, \alpha_{1}, \alpha_{2}, \ldots\right\}$. but the optimization without constraints on the lens pressure leads to the obvious solution given in $\{P\}=\{0,0,1,0, \ldots\}$. This set of parameters corresponds to an initial non-pressurized reflector having perfect parabola shape with zero pressure in the lens and in the torus. This is obviously a trivial result. Indeed, the membrane is characterized by very small bending stiffness. So it is necessary to pressurize slightly the structure in order to maintain a minimal stiffness.

We have tested different numbers of shape parameters. Three parameters have been used in the first analysis: $\{\alpha\}=\alpha_{1}, \alpha_{2}, \alpha_{3}$. We added a fourth one $\alpha_{4}$ in the second analysis. This point has been discussed in Section 5.

The solution proposed here has been to impose the pressure in the lens at the value of $30 \mathrm{~Pa}$. It has been specified thanks to preliminary finite element analysis (see Fig. 4) performed with $\alpha_{1}=1, \alpha_{2}=0, \alpha_{3}=0, p_{l}=30 \mathrm{~Pa}$. Only the lens was considered and the vertex on the lens perimeter was clamped.

This pressure level allows to obtain an acceptable von Mises stress in the membrane reflector inferior to 6.5 MPa. So the optimizing parameters set become

$\{p\}=\left\{p_{t}, \alpha_{1}, \alpha_{2}, \alpha_{3}\right\}$

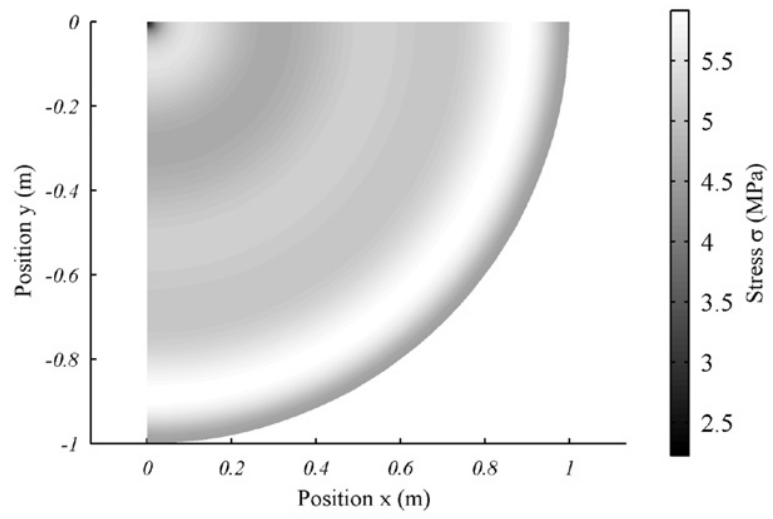

Fig. 4. von Mises stresses in the lens for $\alpha_{1}=1, \alpha_{2}=0, \alpha_{3}=0, p_{l}=30 \mathrm{~Pa}$.

\section{Finite element analysis}

The deformed shape used to evaluate the RMSE function is computed using a finite element analysis based on the energy minimization principle.

The total potential $\Pi$ energy of the inflatable structure is the sum of the internal energy of the body $\Pi^{\text {int }}$ and the external potential energy $\Pi^{\text {ext }}$

$\Pi=\Pi^{i n t}+\Pi^{e x t}$

The expressions of the two energies are discretized using the three nodes true membrane element depicted in Fig. 5. The nodal coordinates $\vec{X}_{i}$ and the material basis vectors $\overrightarrow{A_{i}}$ refer to the initial configuration while $\overrightarrow{x_{i}}$ and $\overrightarrow{a_{i}}$ are the corresponding nodal coordinates and material basis vectors in the current configuration.

\subsection{Discretization of internal potential energy}

The internal potential energy is

$\Pi^{i n t}=\int_{V_{0}} \psi d V_{0}$

$V_{0}$ is the initial volume of the body and $\psi$ the strain energy density.

The material is assumed elastic and governed by a quadratic potential of strain tensor $\overline{\bar{E}}$ such as

$\psi=\frac{\lambda}{2}(\operatorname{tr} \overline{\bar{E}})^{2}+\mu \overline{\bar{E}}: \overline{\bar{E}}$

This is the classical expression of the Saint-Venant Kirchhoff model where $\overline{\bar{E}}$ refers to the Green Lagrange strain tensor while $\lambda$ and $\mu$ are the Lamé constants.

The components of $\overline{\bar{E}}$ are

$E_{\alpha \beta}=\frac{1}{2}\left(\overrightarrow{a_{\alpha}} \cdot \overrightarrow{a_{\beta}}-\overrightarrow{A_{\alpha}} \cdot \overrightarrow{A_{\beta}}\right)$

where Greek indices take the values 1 or 2 . We can see that the strain tensor is constant over a triangular finite element.

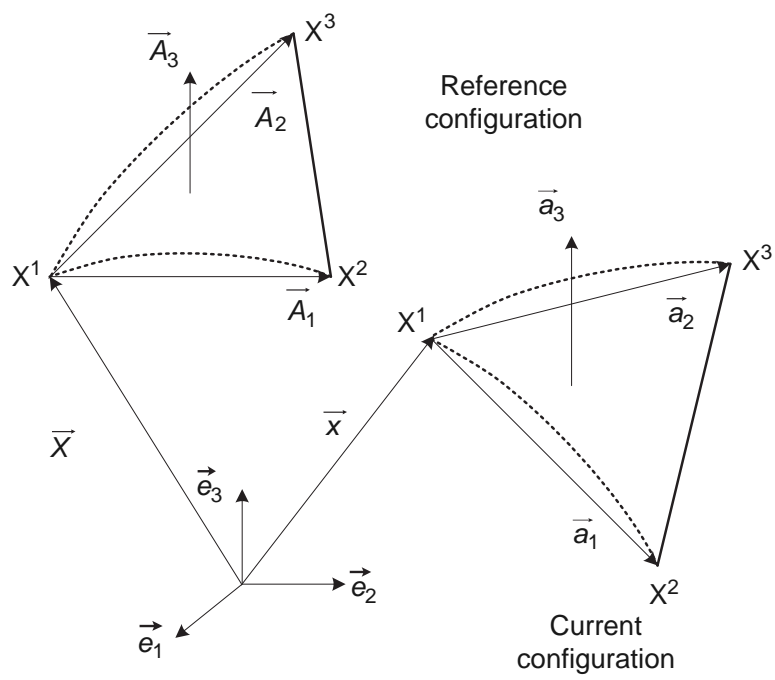

Fig. 5. Triangular finite element discretization of the membrane. 
As shown in Fig. 5, the material basis vectors on the mid-surface are computed with the help of reference $X_{i}^{j}$ and deformed $x_{i}^{j}$ vertices coordinates of triangular finite elements such as

$\overrightarrow{A_{1}}=\overrightarrow{X_{2}}-\overrightarrow{X_{1}}, \quad \overrightarrow{a_{1}}=\overrightarrow{x_{2}}-\overrightarrow{x_{1}}$

$\overrightarrow{A_{2}}=\overrightarrow{X_{3}}-\overrightarrow{X_{1}}, \quad \overrightarrow{a_{2}}=\overrightarrow{x_{3}}-\overrightarrow{x_{1}}$

The internal energy of the structure is obtained by a summation over the $e$ elements

$\Pi^{i n t}=\sum_{e} V_{0} \psi^{e}$

\subsection{Discretization of external potential energy} by

The potential energy of the applied pressure is given

$\Pi^{e x t}=-P V$

where $P$ is the assumed constant internal pressure, and $V$ is the actual volume of the pressurized body. The expression (11) takes a discrete form as a summation over the finite elements

$\Pi^{e x t}=-P \sum_{e}\left(\overrightarrow{a_{1}} \wedge \overrightarrow{a_{2}}\right) \cdot \vec{e}_{3} \sum_{i=1}^{3} \frac{\vec{x}_{i} \cdot \vec{e}_{3}}{3}$

$\vec{e}_{3}$ is the unit vector along the $Z$-axis.

\subsection{Minimization of the total potential energy using conjugate gradient method}

The nonlinear finite element formulation presented here accounts large displacement, large rotation and follower pressures. To be efficient especially in the case of a membrane shape optimization, the solving algorithm has to converge on a bifurcated path even if buckling or wrinkling occurs. Indeed the wrinkling of membrane happens when compressive stress is imposed. It is a really common phenomenon affecting the performance and the reliability of space structure. Nevertheless the wrinkling prediction during a finite element analysis stays a challenging problem.

The energy minimization can be achieved either by firstorder methods, like descent methods, or second-order methods, like quasi-Newtonian ones. The most common approach in finite element analysis is to use second-order methods which require computing the stiffness matrix. This matrix may be ill-conditioned when a significant loss of stiffness occurs. At a bifurcation point, the stiffness matrix is singular and ill-conditioned in their neighborhood. This is why corrective solution methods, like the Newton-Raphson method, may run into difficulties at or near critical points. This ill-conditioning may introduce noise which makes the solution procedure unstable. Sometimes traversing critical points may become computationally overwhelming and may require either specialized techniques or intensive human interventions.

To enable the computational simulation to predict the wrinkle patterns, two methods are classically used. The first one involves the treatment of bifurcation points with asymptotic expansion or a similar process [9]. This method involves the detection of critical points based on the singularity of the tangent stiffness matrix, then the switching on a bifurcated branch. Its detailed developments can be found in [10-12].

The second one is the post buckling analysis [13]. To enable the computational method to predict the wrinkle patterns, an eigenvalue buckling analysis is performed before the calculation. It gives the membrane mode shapes, introduced as geometrical imperfections in the post buckling analysis. Several recent computational studies have employed the post buckling analysis with geometrically non-linear shell finite element models, see [13-15]

Here the simulations have been performed by directly minimizing the total potential energy using a first-order descent method, instead of satisfying the equilibrium equations as done with the usual finite element method. It proves to be an attractive alternative which is particularly efficient for thin structures. Indeed, the proposed solving procedure has been first validated on the Hencky's problem in [16]. Then the ability of the solving procedure to predict wrinkling has been discussed in [17] through the study of two well-known problems: the wrinkling prediction of rectangular membrane under transverse inplane displacements and the wrinkling prediction in a square membrane under corner loads. The numerical developments are implemented in the Surface Evolver code developed by K. Brakke and presented in [18]. A sample of bifurcated solution obtained for the parabolic reflector is shown in Fig. 6. In this sample, the boundary conditions are completely free. Rigid body displacements are correctly handled by the solver used here which is based on the conjugate gradient method.

The main interest of the minimization algorithm is that no specific buckling analysis is carried out during the calculation.

The first-order minimization algorithm used here is a classical conjugate gradient algorithm proposed by Fletcher and Reeves in [19]. It is based on the descent method. Nevertheless, we insist on the fact that the

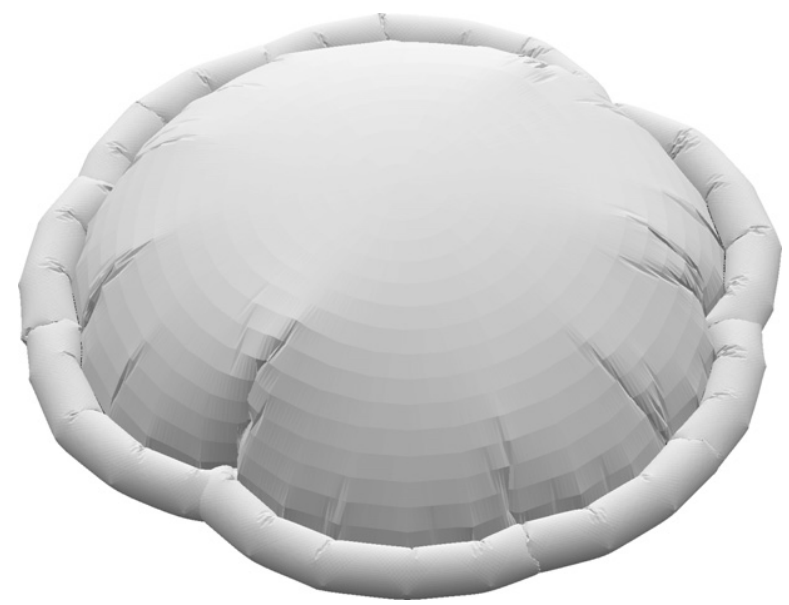

Fig. 6. A sample of bifurcated solution computed using conjugate gradient algorithm. 
gradient of the total potential, which gives the opposite descent direction, must be exactly computed. Thus, it is necessary to provide the analytical expression of the potential gradient in order to obtain the sufficient accuracy and to correctly handle some phenomena like bifurcation. The nine components of the finite element energy gradient are the partial derivatives of $\Pi$ with respect to the coordinates of the vertices $x_{i}^{j}$ i.e.

$(\nabla \Pi)_{i j}=\frac{\partial \Pi}{\partial x_{i}^{j}}$

This is the exact formulation of the gradient of the membrane strain energy used for large scale problems.

The first-order methods were used to seek for a minimum by successive line search. The descent direction is computed thanks to the gradient which gives the direction of greatest increase of $\Pi$. The global convergence of the conjugate gradient algorithm is demonstrated in the case of convex potentials. In wrinkling problems, the potential is non-convex and the algorithm converges toward an existing minimum which may be either a local or a global minimum. Further details about this subject will be found in [20]. The main asset of first-order methods is that they allow to converge toward a local or global minimum even in the neighborhood of a bifurcation point.

The reader must distinguish the minimization of the total potential energy which aims to solve the direct problem - finding the pressurized configuration - and the minimization of the RMSE function which aims to seek for the optimal optimizing parameters. These two minimizations processes are algorithmically nested.

\section{Discussion and results}

Before presenting the design optimization of a space inflatable membrane reflector in Section 5.2, the previously described procedure has been first validated on the Hencky's problem for which a semi-analytical solution is given in Section 5.1.

\subsection{Validation of the optimization process}

Hencky [6] considers the inflation of a circular membrane of initial radius $R$ and thickness $h$, clamped on its rim and subjected to a lateral pressure $P_{m}$. We used the semi-analytical Hencky's solution to validate of the optimizing analysis proposed here. For this purpose, two optimization procedures have been performed:

- The first one aims to find the inflation pressure $P_{m}$ of the lens surface by minimizing the gap between the Fichter's model and the FE model.

- The second one aims to provide the Young Modulus $E$ and the Poisson ratio $v$ by minimizing the gap between the Fichter's model and the FE model for fixed pressure.

\subsubsection{The Fichter's semi-analytical solution}

Analytical solutions for circular membranes made in incompressible isotropic materials can be found in $[21,22]$. For compressible materials, analytical solutions are rather few since the absence of isochoric constraints leads to more complicated kinematics. A review of solution strategies for compressible isotropic materials was presented by Horgan [23, Chapter 4]. When solving a circular membrane, Fichter [24] dropped some secondorder terms in the Green strain components and considered the pressure as a dead load. These approximations led to a simplified solution which is chosen here for comparison in moderate rotations. Let us summarize Fichter's semi-analytical solution given in [24]. The deflection $z_{t h}$ is searched in the form of a power series

$z_{t h}(\rho)=R\left(\frac{P_{m} R}{E h}\right)^{1 / 3} \sum_{0}^{\infty} a_{2 n}\left(1-\rho^{2 n+2}\right)$

By replacing this expression in the equilibrium equation, one obtains the circumferential stress $\Sigma_{\theta \theta}$

$\Sigma_{\theta \theta}(\rho)=\frac{E}{4}\left(\frac{P_{m} R}{E h}\right)^{2 / 3} \sum_{0}^{\infty}(2 n+1) b_{2 n} \rho^{2 n}$

Taking into account the boundary condition $z(R)=0$ enables one to determine the coefficients $a_{2 n}$ and $b_{2 n}$. The values up to $n=10$ are given in [24]

$$
\begin{aligned}
& b_{2}=-\frac{1}{b_{0}^{2}}, \quad b_{4}=-\frac{2}{3 b_{0}^{5}}, \quad b_{6}=-\frac{13}{18 b_{0}^{8}} \\
& b_{8}=-\frac{17}{18 b_{0}^{11}}, \quad b_{10}=-\frac{37}{27 b_{0}^{14}}, \quad b_{12}=-\frac{1205}{567 b_{0}^{17}} \\
& b_{14}=-\frac{219,241}{63,504 b_{0}^{20}}, \quad b_{16}=-\frac{6,634,069}{1,143,072 b_{0}^{23}}, \\
& b_{18}=-\frac{51,523,763}{5,143,824 b_{0}^{26}}
\end{aligned}
$$

$b_{20}=-\frac{998,796,305}{56,582,064 b_{0}^{29}}$

and

$$
\begin{aligned}
& a_{0}=\frac{1}{b_{0}^{1}}, \quad a_{2}=\frac{1}{2 b_{0}^{4}}, \quad a_{4}=\frac{5}{9 b_{0}^{7}} \\
& a_{6}=\frac{55}{72 b_{0}^{10}}, \quad a_{8}=\frac{7}{6 b_{0}^{13}} \quad a_{10}=\frac{205}{108 b_{0}^{16}} \\
& a_{12}=\frac{17,051}{5292 b_{0}^{19}}, \quad a_{14}=\frac{2,864,485}{508,032 b_{0}^{22}}, \quad a_{16}=\frac{103,863,265}{10,287,648 b_{0}^{25}} \\
& a_{18}=\frac{27,047,983}{1,469,664 b_{0}^{28}}, \quad a_{20}=\frac{42,367,613,873}{1,244,805,408 b_{0}^{31}}
\end{aligned}
$$

It should be noted that all the coefficients depend on $b_{0}$ By using the boundary condition $z(R)=0$, Fichter showed that $b_{0}$ is related to the Poisson's ratio by the following equation:

$(1-v) b_{0}+(3-v) b_{2}+(5-v) b_{4}+(7-v) b_{6}+\cdots=0$

It follows that all the other coefficients also depend on the Poisson's ratio only. 


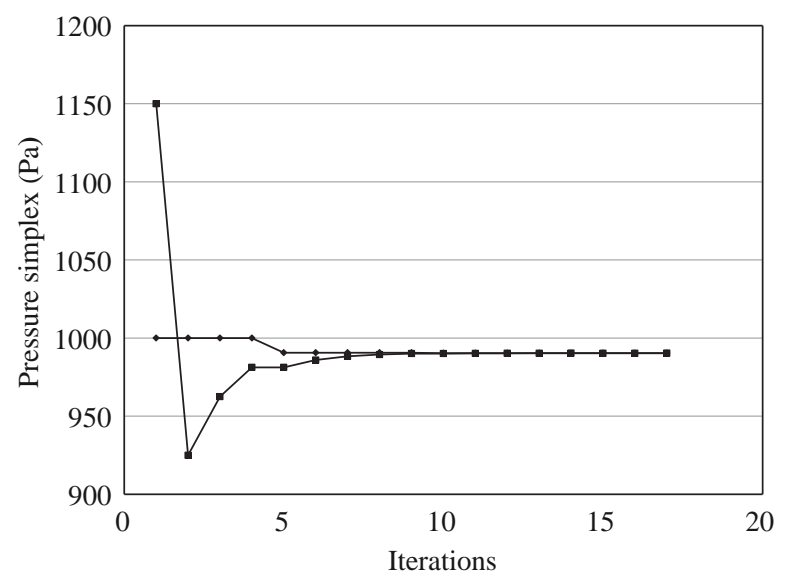

Fig. 7. Pressure evolution during the optimization process.

\subsubsection{Validation 1: pressure optimization}

A circular membrane initially flat of radius $R=1 \mathrm{~m}$ and thickness $h=125 \mu \mathrm{m}$ is considered. Its material data are $E=6 \mathrm{GPa}$ and $v=0.3$. The inflation pressure chosen to compute the Fichter semi-analytical solution $z_{\text {th }}$ is $P_{m}=1000 \mathrm{~Pa}$. It is also the target value we expect to obtain using the optimization process. A mesh of 2048 triangular membrane elements was used to model the whole structure. The minimization procedure of the RMSE function has been initialized using a two vertex simplex where: vertex $1: P_{m}=1150 \mathrm{~Pa}$ and vertex 2 : $P_{m}=1000 \mathrm{~Pa}$. The evolution of the vertex values is given in Fig. 7.

The convergence of the numerical optimization is reached after 17 iterations. This test shows the efficiency of the proposed algorithm since the difference between the computed pressure (990.3 Pa) and the target value of $P_{m}$ is less than $1 \%$.

\subsubsection{Validation 2: material data optimization}

A second validation test has been performed on the circular membrane depicted in Section 5.1.2. It has been discretized with the same number of triangular finite elements. Here, the internal pressure and the geometry have been imposed. The Fichter's semi analytical solution is computed considering the following material data: $E=6 \mathrm{GPa}$ and $v=0.3$. These are the target values we expect to obtain using the optimization procedure. The minimization procedure of the RMSE function has been initialized using the simplex given in Table 2.

The convergence history of the values of $E$ and $v$ are respectively depicted in Figs. 8 and 9.

The optimization of the parameter $E$ gives a value of $5.74 \mathrm{GPa}$. This is close (error of $4.3 \%$ ) of the value chosen to compute the reference solution. Nevertheless a noticeable difference of $13.3 \%$ is observed between the optimized value of $v(0.34)$ and the reference one (0.3). This gap is partially caused by the difference of formulation between the semi-analytical and the numerical model. The coefficients $a_{i}$ and $b_{i}$ used to compute the semianalytical solution depend only on the Poisson ratio. The restriction on the order of polynomial series consequently
Table 2

The initial simplex used for the material data optimization.

\begin{tabular}{lcl}
\hline Vertex & $E(\mathrm{GPa})$ & $v$ \\
\hline Vertex 1 & 8 & 0.6 \\
Vertex 2 & 10.6 & 0.4 \\
Vertex 3 & 13.5 & 0.65 \\
\hline
\end{tabular}

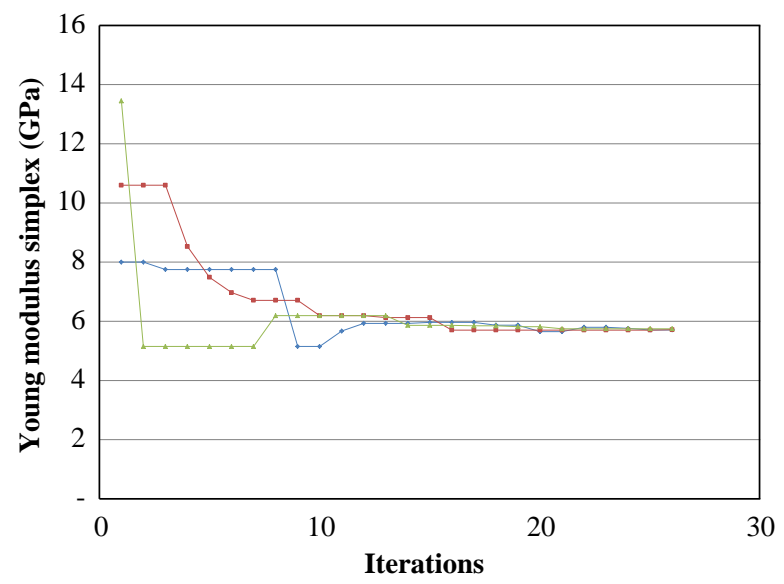

Fig. 8. Evolution of the Young Modulus value during the optimization process.

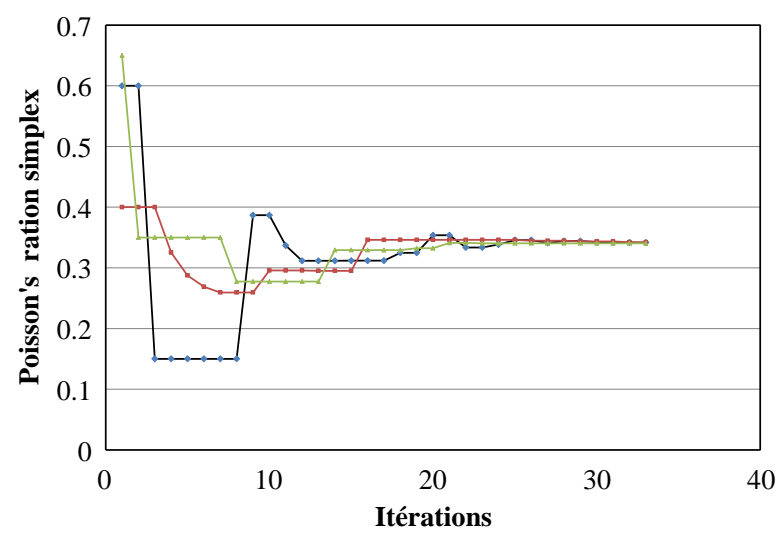

Fig. 9. Evolution of the Poisson's ratio value during the optimization process.

causes an error which affects the value of the Poisson's ratio. This discrepancy comes also from the semi-analytical solution for a circular membrane developed by Fichter, who dropped some second-order terms in the Green strain components. These approximations led to a simplified solution which must be valid in moderate displacements and rotations.

The two previous tests show, thanks to comparison with the semi-analytical solution, the ability of the proposed algorithm to converge toward an acceptable set of parameters in the case of nonlinear membrane optimization problems. Consequently we expect to obtain some good optimization results in more complicated case as the optimization of the inflatable reflector. 


\subsection{Inflatable reflector optimization}

A mesh of 26,192 of the three-node membrane element has been used to model the whole structure (see Fig. 10). A $z$-symmetry condition has been imposed since half structure is represented. In addition, to avoid rigid body displacement in the $x y$-directions, the horizontal displacement of the central node is prescribed to zero.

The optimization study has been performed using three and four shape parameters $\alpha_{i}$. The minimization procedure of the RMSE function has been initialized using the simplex given in Tables 3 and 4 for respectively three and four shape parameters. In the both case, the initial simplex has been chosen thanks to preliminary simulation results.

Then the procedure depicted in Fig. 2 has been performed and the best set of parameters obtained are given in Eqs. (19) and (20)

$$
\begin{aligned}
& \{p\}=\{151.95,0.987,0.038,-0.029\} \\
& \{p\}=\{212 P a, 1.065,-0.280,0.425,-0.211\}
\end{aligned}
$$

These results show that the addition of the parameter $\alpha_{4}$ disturbs the values obtained with three shape parameters. The value attained for the best set of parameters

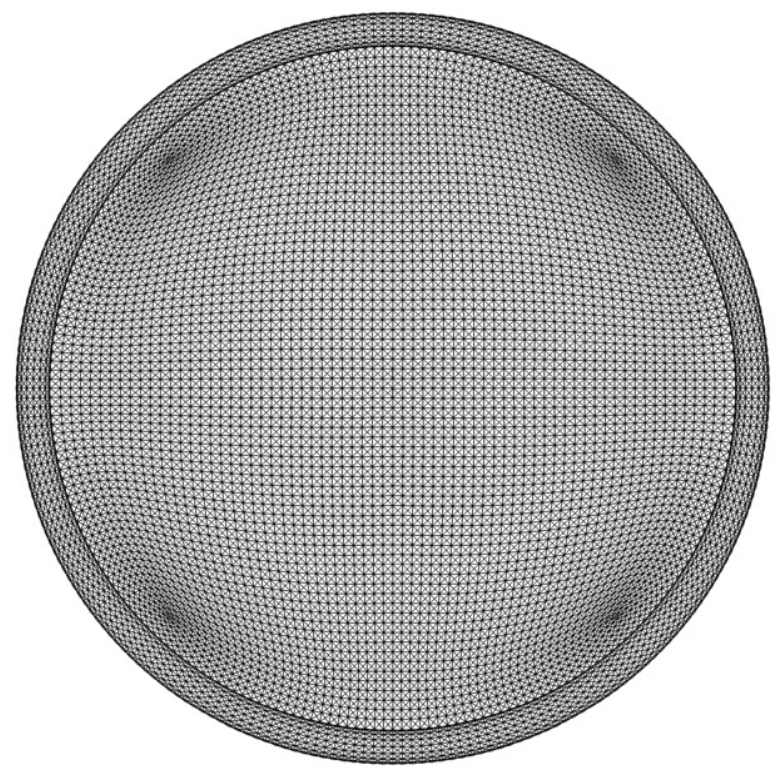

Fig. 10. The finite element model.

Table 3

The initial simplex using three shape parameters.

\begin{tabular}{lllll}
\hline Vertex & $P_{t}(\mathrm{~Pa})$ & $\alpha_{1}$ & $\alpha_{2}$ & $\alpha_{3}$ \\
\hline Vertex 1 & 200 & 0.98 & 0.03 & -0.02 \\
Vertex 2 & 250 & 0.98 & 0.03 & -0.02 \\
Vertex 3 & 200 & 0.95 & 0.03 & -0.02 \\
Vertex 4 & 200 & 0.98 & 0.05 & -0.02 \\
Vertex 5 & 200 & 0.98 & 0.03 & -0.04 \\
\hline
\end{tabular}

Table 4

The initial simplex using four shape parameters.

\begin{tabular}{llllll}
\hline Vertex & $P_{t}(\mathrm{~Pa})$ & $\alpha_{1}$ & $\alpha_{2}$ & $\alpha_{3}$ & $\alpha_{4}$ \\
\hline Vertex 1 & 200 & 0.98 & 0.03 & -0.02 & -0.02 \\
Vertex 2 & 250 & 0.98 & 0.03 & -0.02 & -0.02 \\
Vertex 3 & 200 & 0.95 & 0.03 & -0.02 & -0.02 \\
Vertex 4 & 200 & 0.98 & 0.05 & -0.02 & -0.02 \\
Vertex 5 & 200 & 0.98 & 0.03 & -0.04 & -0.02 \\
Vertex 6 & 200 & 0.98 & 0.03 & -0.02 & -0.04 \\
\hline
\end{tabular}

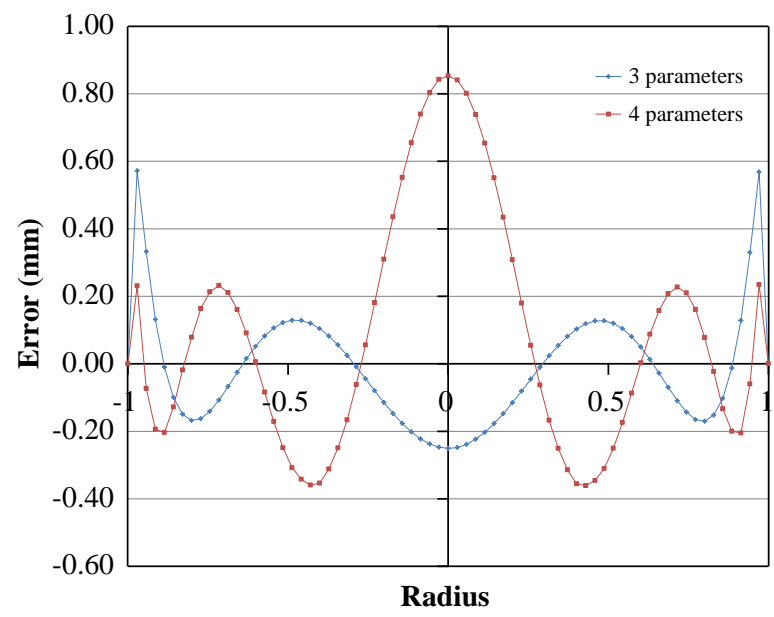

Fig. 11. Gap between the target shape and the effective shapes attained for three and four parameters.

seems therefore to be dependent on the number of shape parameters used. This can be caused by the parameterization chosen to describe the neutral shape. The shape function basis $f_{i}(\rho)=\left(1-\rho^{2 i}\right), i=1, \ldots, n$, presents a lack of orthogonality in the sense that $f_{i}(\rho) \approx f_{i+1}(\rho)$ when $i \rightarrow \infty$. With the polynomial series presented here, the convergence would not be reached on the shape parameters values. However the deviation of the optimized shape relative to the target shape still very acceptable to both cases as we can see in Fig. 11. For this set of parameters, the gap between the target function and the actual shape is given in Fig. 11. The RMSE evaluated for the whole surface is about $175 \mu \mathrm{m}$ for the two cases.

We could expect to improve the solution using a more adequate shape functions and large number of parameters.

The comparison between the two initial shapes, corresponding to the best sets of parameters with three and four parameters, shows that they are close to each others, even if the values of shape parameters are different (see Fig. 12). Lastly, Fig. 13 shows the evolution of the cost function simplex vs the iteration number.

In the both studies it is also possible that best set of parameters exist because the simplex algorithm can converge toward a local minimum of the RMSE function. To avoid this matter, the use of a genetic algorithm (such as proposed in [25] to study a similar problem) may improve the procedure. 


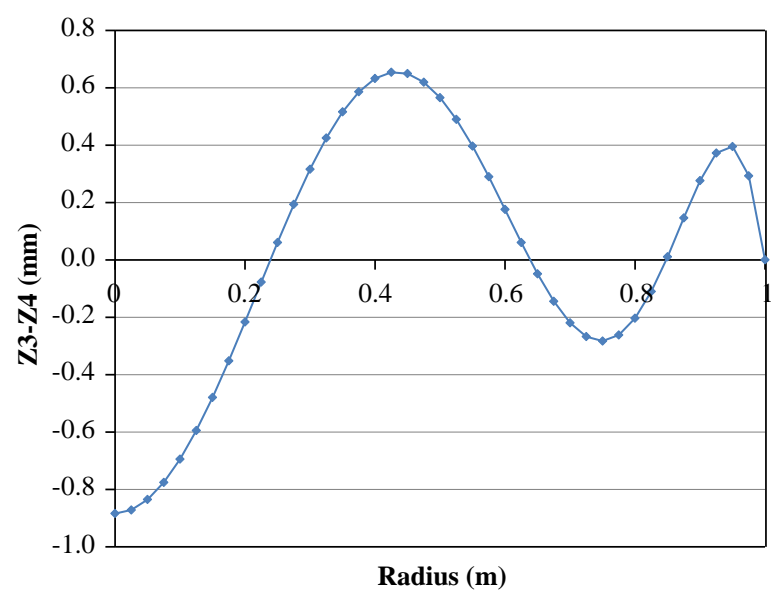

Fig. 12. Gap between the initial shapes corresponding to the optimal parameters (three and four shape parameters).

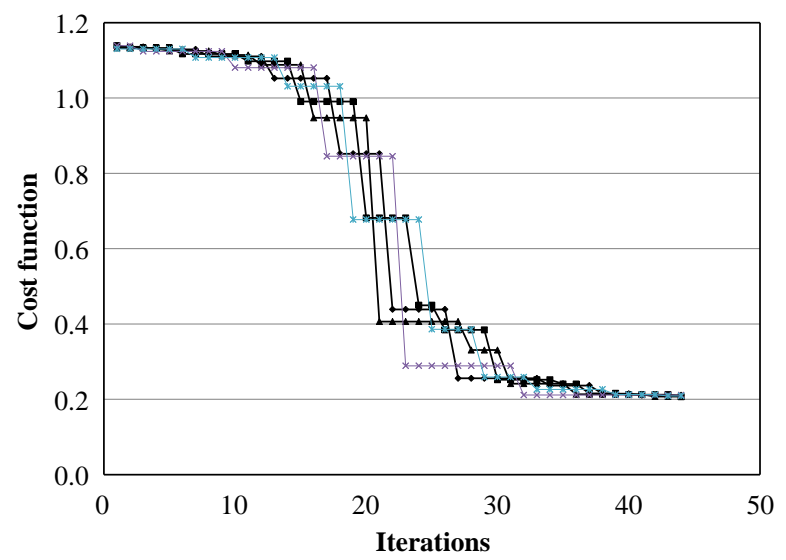

Fig. 13. Evolution of the cost function during the optimization process with three shape parameters.

\section{Conclusion}

This study presents a design tool of a parabolic reflector made of inflatable lens supported by torus. The optimization is done with a constant pressure of $30 \mathrm{~Pa}$ in the lens. We seeked for the initial shape and pressure in the torus that would permit to obtain a perfect parabola.

The neutral shape was defined thanks to a polynomial odd power series expansion. The optimization consisted in finding the first values of the power series and the torus pressure. The direct problem is solved by a non linear finite element analysis to compute the deformed shape of the structure. The RMSE between the deformed shape and the perfect parabola represents the cost function that should be minimized by using the Nedler-Mead simplex algorithm. The difficulty of this optimization is due to the complex highly non-linear direct problem. In thin films, wrinkling may occur which affects the shape of the reflector. To be efficient in a design optimization issue, the resolution algorithm of the direct problem has to achieve the convergence automatically, even though it is on a bifurcated path. In this paper the computation was performed using the conjugate gradient algorithm which proves to be robust. The proposed algorithm has been firstly tested on the inflation of initial flat parabolic structure for which a semi-analytical solution exists. Then the influence of the chosen parameterization is discussed. The results of the optimization show to be very satisfactory, so that the space industry could take advantage of this tool to design inflatable space structures.

\section{Acknowledgments}

The authors thank K. Brakke for his helpful code "Surface Evolver" and C. Talec for useful corrections.

\section{References}

[1] C. Jenkins, Gossamer Spacecraft: Membrane and Inflatable Structures Technology for Space Applications, AIAA, 2001.

[2] J.C. Mankins, A fresh look at space solar power: new architectures, concepts, and technologies, Acta Astronaut. 41 (4-10) (1997) 347-359 (Developing Business).

[3] R.E. Freeland, G.D. Bilyeu, G.R. Veal, Validation of a unique concept for a low-cost, lightweight space-deployable antenna structure, Acta Astronaut. 35 (9-11) (1995) 565-572, doi:10.1016/00945765(95)00018-U (Challenges of Space for a Better World).

[4] R. Freeland, G. Bilyeu, G. Veal, Development of flight hardware for a large, inflatable-deployable antenna experiment, Acta Astronaut. 38 (4-8) (1996) 251-260, doi:10.1016/0094-5765(96)00030-6 (Benefits of Space for Humanity).

[5] L. Palisoc, Y. Huang, Design tool for inflatable space structures, AIAA AIM-974 378 (1997) 2922-2930.

[6] H. Hencky, On the stress state in circular plates with vanishing bending stiffness, Zeitschrift fur Mathematik und Physik 63, 1915.

[7] J.D. Campbell, On the theory of initially tensioned circular membranes subjected to uniform pressure, Quart. J. Mech. Appl. Math. 9 (1956) 84-93.

[8] J. Nelder, R. Mead, A simplex method for function minimization, Comput. J. (1965) 308-313.

[9] A. Diaby, A.L. van, C. Wielgosz, Buckling and wrinkling of prestressed membranes, Finite Elem. Anal. Des. 42 (11) (2006) 992-1001, doi:10.1016/j.finel.2006.03.003.

[10] E. Riks, An incremental approach to the solution of snapping and buckling problems, Int. J. Solids Struct. 15 (7) (1979) 529-551.

[11] M. Crisfield, Non-Linear Finite Element Analysis of Solids and Structures: Advanced Topics, John Wiley \& Sons, Inc., New York, NY, USA, 1997.

[12] A. Magnusson, Treatment of bifurcation points with asymptotic expansion, Comput. Struct. 77 (5) (2000) 475-484.

[13] Y. Wong, S. Pellegrino, Wrinkled membranes. Part iii: numerical simulations, J. Mech. Mater. Struct. 1 (2006) 61-93.

[14] C. Wang, H. Tan, X. Du, Z. Wan, Wrinkling prediction of rectangular shell-membrane under transverse in-plane displacement, Int. J. Solids Struct. 44 (20) (2007) 6507-6516, doi:10.1016/ j.ijsolstr.2007.02.036.

[15] Y. Lecieux, R. Bouzidi, Experimental analysis on membrane wrinkling under biaxial load-comparison with bifurcation analysis, Int. J. Solids Struct. 47 (18-19) (2010) 2459-2475.

[16] R. Bouzidi, A.L. van, Numerical solution of hyperelastic membranes by energy minimization, Comput. Struct. $82(23-26)$ (2004) 1961-1969, doi:10.1016/j.compstruc.2004.03.057 (Computational Structures Technology).

[17] Y. Lecieux, R. Bouzidi, Numerical wrinkling prediction in thin hyperelastic structures by direct energy minimization, in: Proceedings of the CST 2010, 2010.

[18] K.A. Brakke, The surface evolver, Exp. Math. 1 (1992) 141-165.

[19] R. Fletcher, C.M. Reeves, Function minimization by conjugate gradients, Comput. J. 7 (2) (1964) 149-154, doi:10.1093/comjnl/ 7.2.149.

[20] L. Grippo, S. Lucidi, A globally convergent version of the polakribière conjugate gradient method, Math. Program. 78 (3) (1997) 375-391. 
[21] R. Ogden, Large deformation isotropic elasticity: on the correlation of theory and experiment for incompressible rubber like solids, Proc. R. Soc. Lond. A 326 (1972) 565-584.

[22] E. de Souza Neto, D. Peric, D. Owen, Finite elasticity in spatial description: linearization aspects with $3 \mathrm{~d}$ membrane applications, Int. J. Num. Meth. Eng. 38 (1995) 3365-3381.

[23] Y. Fu, R. Ogden, Nonlinear Elasticity. Theory and Applications, Cambridge University Press, 2001.
[24] W. Fichter, Some Solutions for the Large Deflections of Uniformly Loaded Circular Membranes, NASA Technical Paper 3658-NASA Langley Research Center, Hampton, VA, 1997.

[25] R. Orszulik, J. Shan, M. Stachowsky, Membrane structure active flatness control using genetic algorithm with online objective reweighting, Acta Astronaut. 68 (11-12) (2011) 2012-2024. 